\author{
Alterstice \\ Revue internationale de la recherche interculturelle \\ International Journal of Intercultural Research \\ Revista International de la Investigacion Intercultural
}

\title{
Faible participation des travailleurs immigrants aux mesures de santé et sécurité au travail dans les petites entreprises
}

\section{Sylvie Gravel, Gabrielle Legendre et Jacques Rhéaume}

Volume 2, numéro 2, 2012

Regards pluriels sur les interventions sociales et de santé en contexte de diversité

URI : https://id.erudit.org/iderudit/1077566ar

DOI : https://doi.org/10.7202/1077566ar

Aller au sommaire du numéro

Éditeur(s)

Alterstice

ISSN

1923-919X (numérique)

Découvrir la revue

Citer cet article

Gravel, S., Legendre, G. \& Rhéaume, J. (2012). Faible participation des travailleurs immigrants aux mesures de santé et sécurité au travail dans les petites entreprises. Alterstice, 2(2), 63-78. https://doi.org/10.7202/1077566ar

\section{Résumé de l'article}

Une analyse de la participation des travailleurs aux mesures préventives a été réalisée dans le cadre d'une étude portant sur la santé et la sécurité au travail (SST) dans les petites entreprises (PE) montréalaises de 10 à 50 employés embauchant des travailleurs immigrants. Comparativement aux travailleurs canadiens d'origine, les travailleurs immigrants bénéficiaient de moins de formations en SST et étaient moins enclins à identifier les risques, à déclarer leurs lésions lorsqu'ils se blessaient et à participer aux enquêtes d'accidents. Plusieurs ne portaient pas d'équipement de protection même si l'employeur en fournissait un. De façon générale, les travailleurs immigrants méconnaissaient leurs droits et leurs obligations en matière de SST. Certaines PE, surtout celles dont les dirigeants étaient diplômés en gestion ou en SST, faisaient des efforts soutenus pour corriger cette méconnaissance par des activités de formation, des systèmes de compagnonnage entre collègues originaires des mêmes pays ou communicant dans la même langue, des actions pertinentes, mais souvent insuffisantes. Les PE qui tentaient de surmonter les écarts de compréhension culturelle et linguistique de la SST étaient plutôt des entreprises hétérogènes sur le plan des origines culturelles et des langues : les dirigeants étaient majoritairement canadiens, alors que les superviseurs et les employés étaient originaires de divers pays. Au contraire, dans les $\mathrm{PE}$ homogènes où les dirigeants, les superviseurs et les travailleurs provenaient des mêmes pays d'origine (ex : Liban, Inde, etc.), les problèmes de compréhension des mesures préventives de SST se posaient à tous les niveaux hiérarchiques. 


\section{ज alterstice \\ ARTICLE THÉMATIQUE \\ Faible participation des travailleurs immigrants aux mesures de santé et sécurité au travail dans les petites entreprises}

Sylvie Gravel ${ }^{1}$, Gabrielle Legendre ${ }^{1}$ et Jacques Rhéaume ${ }^{1}$

\section{Résumé}

Une analyse de la participation des travailleurs aux mesures préventives a été réalisée dans le cadre d'une étude portant sur la santé et la sécurité au travail (SST) dans les petites entreprises (PE) montréalaises de 10 à 50 employés embauchant des travailleurs immigrants. Comparativement aux travailleurs canadiens d'origine, les travailleurs immigrants bénéficiaient de moins de formations en SST et étaient moins enclins à identifier les risques, à déclarer leurs lésions lorsqu'ils se blessaient et à participer aux enquêtes d'accidents. Plusieurs ne portaient pas d'équipement de protection même si l'employeur en fournissait un. De façon générale, les travailleurs immigrants méconnaissaient leurs droits et leurs obligations en matière de SST. Certaines PE, surtout celles dont les dirigeants étaient diplômés en gestion ou en SST, faisaient des efforts soutenus pour corriger cette méconnaissance par des activités de formation, des systèmes de compagnonnage entre collègues originaires des mêmes pays ou communicant dans la même langue, des actions pertinentes, mais souvent insuffisantes. Les PE qui tentaient de surmonter les écarts de compréhension culturelle et linguistique de la SST étaient plutôt des entreprises hétérogènes sur le plan des origines culturelles et des langues : les dirigeants étaient majoritairement canadiens, alors que les superviseurs et les employés étaient originaires de divers pays. Au contraire, dans les PE homogènes où les dirigeants, les superviseurs et les travailleurs provenaient des mêmes pays d'origine (ex: Liban, Inde, etc.), les problèmes de compréhension des mesures préventives de SST se posaient à tous les niveaux hiérarchiques.

\section{Rattachement des auteurs \\ ${ }^{1}$ Université du Québec à Montréal (UQÀM), Montréal, Canada}

\section{Correspondance}

gravel.s@uqam.ca

\section{Mots clés}

santé et sécurité; travailleurs immigrants; participation

\section{Pour citer cet article :}

Gravel, S., Legendre, G. et Réhaume, J. (2012). Faible participation des travailleurs immigrants aux mesures de santé et sécurité au travail dans les petites entreprises. Alterstice, 2(2), 63-78. 


\section{Introduction et contexte}

Depuis la Confédération, le Canada soutien en partie sa croissance économique et démographique grâce à sa politique d'immigration. Au cours des prochaines années, cette politique prévoit de limiter les demandes provenant des travailleurs qualifiés répondants aux critères des professions prioritaires, et d'augmenter le nombre de permis pour l'embauche de travailleurs étrangers temporaires (TÉT) (Citoyenneté et immigration Canada, 2012). Cette tendance est déjà manifeste : 190842 TÉT ont été embauchés en 2011, soit 6,5\% de plus qu'en 2010 (Citoyenneté et immigration Canada, 2012). Au Québec, l'embauche des TÉT est passée de 16775 travailleurs en 2002 à 34369 travailleurs en 2011 (Citoyenneté et Immigration Canada, 2011). C'est une tendance qui, selon la Fédération canadienne des entreprises indépendantes (2011), s'avère une solution à la pénurie de main-d'œuvre dans le secteur manufacturier, et particulièrement pour les petites entreprises (PE, moins de 50 travailleurs) (Demarco et Georgy, 2009; Demarco, McGrath-Gaudet et Hindy, 2007).

Au cours des dernières années, les nouveaux travailleurs admis au Canada, résidents permanents ou temporaires, sont originaires de pays non européens, ce qui modifie considérablement les approches d'intégration. Par ordre d'importance, ils sont originaires des Philippines (14,1\%), de la République populaire de Chine (11,5 \%) et de l'Inde (10\%) (Citoyenneté et immigration Canada, 2012). Les 70000 travailleurs temporaires admis en 2011 - destinés aux emplois saisonniers du secteur agricole - provenaient surtout d'Amérique latine et centrale et des Caraïbes (Citoyenneté et immigration Canada, 2011). Au Québec, en 2011, 625 entreprises du secteur agricole, avicole, de la transformation alimentaire et de l'aménagement paysager ont embauché près de 7000 TÉT, originaires également de ces régions du monde (F.E.R.M.E., 2012).

Si les pays de l'OCDE, dont fait partie le Canada, considèrent l'immigration comme un levier de croissance, tous savent que le plein potentiel de ce levier ne peut s'exercer que si on lutte contre les inégalités d'insertion au marché du travail. Cette lutte passe notamment par la reconnaissance des diplômes et de l'expérience acquise à l'étranger, par la maîtrise des langues officielles et par une meilleure gestion des ressources humaines, dont la gestion de la diversité et la lutte contre la discrimination, étant donné que plusieurs facteurs contribuent à exclure ces travailleurs des emplois les mieux rémunérés et offrant les meilleures conditions de travail (Chicha et Charest, 2008; Forcier et Handal, 2012; Handal, Viens, Hurteau et Hébert, 2012). Une situation à laquelle n'échappe pas le Canada, pays où les immigrants connaissent davantage le chômage et occupent des emplois moins bien rémunérés, accusant un revenu 2,7 fois inférieur à celui des natifs (Forcier et Handal, 2012; Handal, Viens, Hurteau et Hébert, 2012).

Des organismes dédiés à l'intégration ont dressé un profil de l'employabilité des travailleurs immigrants, indiquant que ces travailleurs sont embauchés surtout dans les secteurs manufacturier (31,3\%), de l'hébergement et la restauration (8,8\%), du commerce au détail (8\%) et des services professionnels et techniques $(7,7 \%)$, avec une concentration particulière des hommes dans le secteur du transport et des femmes dans celui de l'éducation (Lacroix et Pipon, 2007). Les employeurs sont généralement des PE, un acteur économique important, puisqu'elles représentaient en 200995 \% des entreprises canadiennes (Statistique Canada, 2009). Concentrées dans les grandes villes, ces PE sont à l'image de l'immigration. Par exemple, en 2008, sur l'île de Montréal, l'une des trois villes canadiennes cosmopolites du Canada, on comptait 7698 PE, dont 29 \% employaient une main-d'œuvre n'ayant pas une connaissance fonctionnelle des langues officielles et 20,3\% étaient administrées par des propriétaires parlant une autre langue que le français et l'anglais (Bouchard, 2008).

Malgré l'évidence de la présence de travailleurs immigrants au sein des PE en milieu urbain, l'échec des processus d'intégration se manifeste par l'absence d'une approche concertée pour l'adaptation culturelle et linguistique des pratiques de SST (Lachance, 2008; Levée, 2011). Il existe certes toute une littérature sur les pratiques de la SST dans les PE (Champoux et Brun, 1999; Eakin, Lamm et Limborg, 2000; Simard, Carpentier-Roy, Marchand et Ouellet, 2002). Mais peu d'études documentent la situation des travailleurs immigrants, parce que les fichiers publics ne disposent pas de variables pouvant distinguer ces travailleurs selon leur origine (Gravel et collab., 2006). Seuls les croisements de fichiers destinés à l'analyse des flux migratoires avec ceux des déclarations des lésions professionnelles ont permis de réaliser ces études (Smith et Mustard, 2009 et 2010; Socialstyrelsen, 1995). 
Certains travaux, dont ceux de Gravel et collab. (2006) et de Premji et collab. (2010), démontrent que la précarité économique et de statut freine les immigrants dans l'exercice de leurs droits en matière de SST. Cela est d'autant plus marqué lorsque les analyses distinguent les conditions de SST selon les statuts de travailleur régulier résident permanent, de travailleur d'agence de location de main-d'œuvre et de TÉT (Henneberry et Preibisch, 2010; Underhill et Quinlan, 2011). Répondant à un besoin de survie économique, les travailleurs immigrants sont contraints d'accepter des emplois qui les exposent à des risques importants de lésions professionnelles (Lachance, 2008). Même les résidents permanents, plus avantagés que les autres catégories de travailleurs, participent peu aux activités préventives et connaissent des difficultés d'accès à l'information et à l'indemnisation (Brown, Domenzain et Villoria-Siegert, 2002; Gravel, Rhéaume et Legendre, 2011; Henshaw, 2002; Kosny et collab., 2012). Ils doivent surmonter certaines barrières, dont la méconnaissance de leurs droits, les difficultés de communication et la crainte de représailles, en particulier celle de perdre leur lien d'emploi ou le droit de parrainer les membres de leur famille (Gravel et collab., 2006).

À ces barrières à la participation des travailleurs immigrants à la culture de la SST s'ajoutent les contraintes de gestion de la petite entreprise (Gravel, Brodeur, Champagne, Lippel et Vissandjée, 2007). Menacées par les fluctuations de l'économie, les PE consacrent peu de temps et de moyens aux pratiques préventives en SST (Eakin, Lamm et Limborg, 2000; Lansdown, Deighan et Brotherton, 2007). Et, parallèlement, elles sont moins assujetties aux mesures de contrôle et à la réglementation, alors même que les sanctions imposées seraient les principales motivations des PE à se conformer à la réglementation (Amodu, 2008; Lansdown, Deighan et Brotherton, 2007; Mendeloff, Nelson, Ko et Haviland, 2006; Quinlan et Mayhew, 2000). Ayant des ressources limitées, les PE utiliseraient plus aisément les services externes gratuits, dont les services en ligne qui ont l'avantage d'être anonymes, plutôt que les services de conseillers (Desmarais, 2004; Health and Safety Executive, 2005; Lansdown, Deighan et Brotherton, 2007).

Malheureusement, c'est trop souvent à la suite d'un événement grave que l'on tente de corriger les lacunes de prévention envers les groupes vulnérables. Confronté au décès tragique de travailleurs immigrants dans le secteur de la construction, un groupe d'experts en SST de l'Ontario a documenté leur accès aux services et aux informations (Kosny et collab., 2011). Leurs recommandations invitaient les divers paliers gouvernementaux à adapter le matériel d'information et de formation aux travailleurs immigrants récents et allophones, à inscrire sur les formulaires de demande d'immigration quelques consignes sur les droits et obligations des travailleurs en matière de SST et à créer des bibliothèques en ligne traitant de SST avec des modules d'information pour les travailleurs en recherche d'emploi (Kosny et collab., 2011; Workers compensation board of Manitoba, 2008).

Bien qu'elles soient intéressantes et pertinentes, ces recommandations ne visent pas les employeurs : elles laissent une fois de plus aux travailleurs la responsabilité de s'initier à des pratiques méconnues et de s'informer, alors que ces derniers sont en processus de recherche d'emploi par survie économique. Compte tenu de l'importance numérique des PE en tant qu'employeurs, compte tenu également du nombre de travailleurs immigrants dans ces PE et étant donné que l'immigration est l'un des leviers de croissance économique, il est primordial d'accorder une attention particulière aux pratiques de SST au sein des PE. Comment accompagner ces PE, principaux employeurs des travailleurs immigrants de tous statuts, afin qu'elles adaptent leurs pratiques de SST?

Au-delà des constats de la précarité des travailleurs immigrants et des moyens restreints des PE, il existe des pratiques, peut-être marginales, mais tout de même réelles de prévention de la SST. Quelles sont ces pratiques? Des aménagements culturels et linguistiques ont-ils été tentés pour surmonter les barrières d'accès décriées dans la littérature? Quelle place occupent les travailleurs immigrants dans cette dynamique de prise en charge des mesures de SST qui se veut paritaire? Sont-ils invités à participer aux diverses étapes d'identification de résolution de problèmes? Disposent-ils des connaissances pertinentes pour s'impliquer?

La présente étude veut contribuer, au-delà de ces constats de précarisation, au développement potentiel d'une approche interculturelle de la SST en identifiant les stratégies favorables à la prise en charge des mesures de santé et sécurité au travail dans les petites entreprises montréalaises embauchant une main-d'œuvre immigrante. 


\section{Méthodologie}

Il s'agit d'une étude prospective, par étude de cas, basée sur un échantillon de 28 PE du secteur privé, sans égard à la présence syndicale, et embauchant entre 10 et 50 travailleurs. Cet échantillon raisonné a été divisé en deux groupes à des fins de comparaison. Le groupe des PE d'observation ( $n=19$ ) est composé d'entreprises ayant une main-d'œuvre composée de $25 \%$ et plus de travailleurs immigrants. À l'inverse, dans le groupe de comparaison $(n=9)$, la main-d'œuvre est composée de moins de $25 \%$ travailleurs immigrants. Les travailleurs immigrants ont divers statuts (citoyen, immigrant reçu, réfugié ou en attente de statut) et leur durée de séjour au Canada est variable, mais nous n'avons inclus aucun travailleur temporaire. La comparaison entre les deux groupes, basée sur le poids démographique des travailleurs immigrants au sein des petites entreprises, visait au départ à cerner la présence de stratégies distinctes de prise en charge de la SST. Or cette distinction s'est avérée moins intéressante pour la compréhension des stratégies de prises en charge de la SST que celle ayant émergé des analyse, à savoir les modalités de composition (hétérogène ou homogène) entre les trois niveaux hiérarchiques (dirigeants, superviseurs et travailleurs) au sein des entreprises.

En 2007, au moment de la conception de cette étude, les entreprises de l'échantillon œuvraient sur un territoire montréalais très cosmopolite, dont le point commun était d'être desservi par les équipes de santé au travail (médecins, infirmières, hygiéniste industriel, etc.) des partenaires à l'étude, soit les Centres des services sociaux et de santé de la Montagne (CSSS) et de l'Ouest-de-I'Île, des établissements de santé publique ayant le mandat d'accompagnement gratuit des entreprises dont les activités économiques étaient jugées à risque. À cette époque, sur le seul territoire du CSSS de la Montagne, 14903 entreprises étaient recensées, dont 787 dans le groupe prioritaire en fonction des critères établis par la Commission de santé et de sécurité au travail (CSST), l'instance publique de surveillance provinciale au Québec. Parmi ces 14903 entreprises, 89 \% comptaient 50 travailleurs et moins. Outre les critères de taille (10-50 travailleurs), les PE de l'échantillon devaient avoir bénéficié d'une intervention de l'équipe de professionnels de santé au travail (SAT) des CSSS de la Montagne et de l'Ouest-de-l'île entre juin 2008 et juin 2010. Sur les 36 entreprises sollicitées par l'intermédiaire des professionnels de SAT des deux CSSS, 28 ont accepté de participer, soit un taux de participation de $78 \%$. Les autres ont invoqué un manque de temps pour nous accorder un entretien.

La grille d'entrevue était préalablement envoyée par courriel aux répondants de SST des PE afin de les guider sur les informations recherchées. La répartition des PE entre le groupe d'observation et le groupe de comparaison n'a été possible qu'à la suite des entretiens menés auprès des répondants de SST des PE, puisqu'aucune donnée administrative consignée par les autorités de SST n'indiquait la composition de la main-d'œuvre selon les critères choisis pour l'enquête (en fonction des pays de naissance, de la durée de séjour au Canada, etc.). Le recrutement des entreprises pour le groupe de comparaison a été plus long que prévu, faute d'entreprises ayant embauché plus de $75 \%$ de travailleurs nés au Canada.

Trois sources de données ont été exploitées : a) les entretiens avec les répondants (dirigeants et gestionnaires) de la SST des PE de notre échantillon $(n=28)$; b) les entretiens avec des professionnels de la SAT qui œuvrent dans ces PE $(n=26)$ et $c)$ les questionnaires autoadministrés par les travailleurs de ces PE $(n=181)$. Les entretiens avec les répondants, de type semi-dirigé et d'une durée d'une heure, ont été menés en anglais ou en français sur les lieux de travail par les chercheurs (auteurs de cet article) accompagnés d'un assistant de recherche. Les entretiens semidirigés avec les professionnels de SAT des CSSS se sont également déroulés sur les lieux de travail. Les questions abordaient sensiblement les mêmes thèmes, avec une insistance sur la réaction des entreprises aux recommandations et aux conseils prodigués. La période de collecte de données de ces deux sources a été de novembre 2008 à juillet 2010. La troisième source de données a été le questionnaire autoadministré par les travailleurs. La sollicitation s'est faite auprès des répondants de la SST des 28 PE participantes entre septembre 2010 et janvier 2011. Le questionnaire à choix de réponses multiples, complété en 12 à 15 minutes, était disponible en quatre langues : français, anglais, espagnol et arabe. En fonction des besoins identifiés par le répondant de la SST de l'entreprise, les questionnaires dans les diverses versions étaient acheminés par courrier aux PE participantes (19/28). Ce questionnaire, qui n'offrait aucune possibilité d'associer le travailleur-répondant à son entreprise, était accompagné d’une enveloppe préaffranchie destinée à l'équipe de recherche. Tous les travailleurs 
qui ont participé ont reçu deux billets d'entrée au cinéma, cadeau acheminé par la poste grâce à une liste distincte obtenue par le biais des questionnaires complétés.

Les thèmes abordés étaient sensiblement les mêmes dans les trois sources de données: le comité de SST, la formation et l'information, les arguments favorables et défavorables à la SST, les compétences et les moyens disponibles en SST, les consignes de sécurité, les mesures d'urgence, les équipements de protection individuelle et l'entretien des équipements et des machines. Les données ont été traitées sur les logiciels Excel, Nvivo 8 et SPSS. L'analyse de la participation des travailleurs immigrants recoupe transversalement l'ensemble de ces thèmes.

Parmi les 28 PE étudiées, 7 se sont distinguées par leurs pratiques intéressantes, voir même exemplaires, pour favoriser la participation des travailleurs immigrants comme de l'ensemble des travailleurs. Une seconde entrevue a été conduite afin d'obtenir des précisions sur ces pratiques exemplaires.

Mentionnons que tous les travaux entourant la réalisation de la recherche (stratégie de recherche, échantillon, sollicitation, grille d'entrevue, etc.) ont été discutés avec un comité de suivi composé de relayeurs pour le transfert des connaissances vers les communautés de pratique. Ce comité regroupait des personnes issues de la recherche en SST et des conseillers des mutuelles de prévention, des représentants patronaux de la PE et des représentants des services destinés aux immigrants, travailleurs et employeurs. Ce comité a été initié et animé par les services à la valorisation des résultats de la recherche du bailleur de fonds, l'Institut de recherche Robert-Sauvé en Santé et Sécurité au Travail (IRSST).

\section{Résultats}

Les résultats de cette étude, présentés selon la source des données, portent essentiellement sur la faible participation des travailleurs aux mesures de SST. Les principaux thèmes explorés dans cette section sont

- le portrait sociodémographique des entreprises étudiées;

- la participation des travailleurs immigrants selon les responsables de SST des PE;

- la participation des travailleurs immigrants selon les professionnels de SAT des CSSS;

- la participation des travailleurs immigrants selon les travailleurs;

- les pratiques exemplaires pour inciter les travailleurs immigrants à participer.

\section{Portrait sociodémographique des entreprises étudiées}

Les entreprises participantes emploient en moyenne 38 travailleurs. Parmi ces entreprises, 6 étaient saisonnières avec un nombre d'employés fluctuant entre 35 et 65 selon le cycle de production. Le personnel était majoritairement composé d'hommes, tant chez les travailleurs (78\%) que chez les superviseurs ou contremaîtres (79\%) et les dirigeants (96\%). Le pourcentage d'employés possédant un niveau de scolarisation postsecondaire était assez élevé pour ces trois catégories : $48 \%$ parmi les travailleurs, $62 \%$ parmi les superviseurs et $77 \%$ parmi les dirigeants. L'ancienneté était aussi assez élevée, supérieure à cinq ans pour $59 \%$ des travailleurs, $71 \%$ des superviseurs et $88 \%$ des dirigeants.

La majorité des entreprises participantes (23/28) œuvraient dans des secteurs de production faisant l'objet d'une surveillance : industrie chimique, du caoutchouc et des matières plastiques; fabrication des produits en métal; transformation des métaux et du bois et enfin transport, des secteurs qui embauchent généralement peu de femmes.

Le critère de séparation des PE en deux groupes, à savoir la concentration à plus ou moins $25 \%$ de la main-d'œuvre composée d'immigrants, avait été défini arbitrairement par des experts de SST. Or il s'est avéré que la concentration d'immigrants au sein des PE était plus importante que prévu. Une PE sur deux a une main-d'œuvre composée de plus de $50 \%$ d'immigrants, certaines allant jusqu'à $90 \%$ d’immigrants. Un résultat qui a fait émerger un facteur important dans l'analyse des dynamiques de prise en charge de la SST : la concentration d'immigrants à chacun des trois niveaux hiérarchiques, dirigeants (propriétaires ou directeur général), superviseurs (contremaîtres et chef d'équipe) et travailleurs. Une concentration qui reflète également l'existence des réseaux sociaux 
d'intégration économique sur le marché du travail. Dans notre échantillon, trois configurations ont dominé ces dynamiques :

- les entreprises homogènes où les travailleurs, les superviseurs et les dirigeants étaient issus de l'immigration, voire des mêmes pays;

- les entreprises homogènes canadiennes, où les travailleurs, les superviseurs et les dirigeants étaient canadiens de naissance;

- les entreprises mixtes, où les travailleurs étaient issus de l'immigration - parfois des mêmes pays, parfois de pays différents - et où les superviseurs et les dirigeants étaient majoritairement canadiens de naissance.

Avec un échantillon plus grand de $\mathrm{PE}$, d'autres configurations auraient sans doute émergé. Le tableau 1 présente quelques exemples de PE qui illustrent bien le phénomène des entreprises homogènes.

Tableau 1 Exemples d'entreprises homogènes :

composition de la main-d'œuvre des PE de l'échantillon selon le pays de naissance

\begin{tabular}{|c|c|c|c|c|c|c|}
\hline $\mathbf{N}^{\circ}$ entreprise & \multicolumn{2}{|c|}{ Dirigeants } & \multicolumn{2}{|c|}{ Superviseurs } & \multicolumn{2}{|c|}{ Travailleurs } \\
\hline$n^{\circ} 02$ & Inde : $100 \%$ & (1) & Inde : $50 \%$ & $(1 / 2)$ & Inde : $77 \%$ & $(23 / 30)$ \\
\hline$n^{\circ} 20$ & Afrique : $50 \%$ & $(1 / 2)$ & Haïti : 50 \% & $(2 / 4)$ & $\begin{array}{r}\text { Haïti : } 72 \% \\
\text { Afrique : } 7 \%\end{array}$ & $\begin{array}{c}(10 / 14) \\
(1 / 14)\end{array}$ \\
\hline$n^{\circ} 28$ & Liban : $100 \%$ & $(2 / 2)$ & Liban : $25 \%$ & $(1 / 4)$ & Liban : $21 \%$ & $(7 / 34)$ \\
\hline
\end{tabular}

La moitié des entreprises de l'échantillon (15/28) étaient homogènes, c'est-à-dire que les dirigeants, les superviseurs et les travailleurs étaient issus des mêmes pays d'origine: Inde, Congo, Liban, Roumanie, etc. On comptait également 7 entreprises sur les 28 dont les dirigeants et les superviseurs d'origine canadienne employaient des travailleurs d'origines mixtes (Haïtiens, Coréens, Marocains et Iraniens). Six des 28 entreprises (incluant le groupe de comparaison) embauchaient essentiellement des travailleurs nés au Canada aux trois niveaux hiérarchiques. Cette caractéristique de l'homogénéité et de l'hétérogénéité des origines de la maind'œuvre au sein des entreprises a émergé lors du traitement des données sur les pratiques de SST (initiation à la tâche, formation sur la conduite de chariot élévateur, systèmes d'information sur les matières dangereuses, mesures d'urgence) auxquelles les travailleurs devaient être en principe initiés. Compte tenu du caractère exploratoire de l'étude, cet élément a été retenu comme facteur explicatif des dynamiques de la prise en charge des mesures de SST dans les PE. Ce résultat a également étonné, car plus de la moitié des entreprises (15/28) étaient homogènes « ethniques » avec des immigrants aux trois niveaux hiérarchiques (figure 1).

Figure 1 Répartition des PE selon les origines de la main-d'œuvre

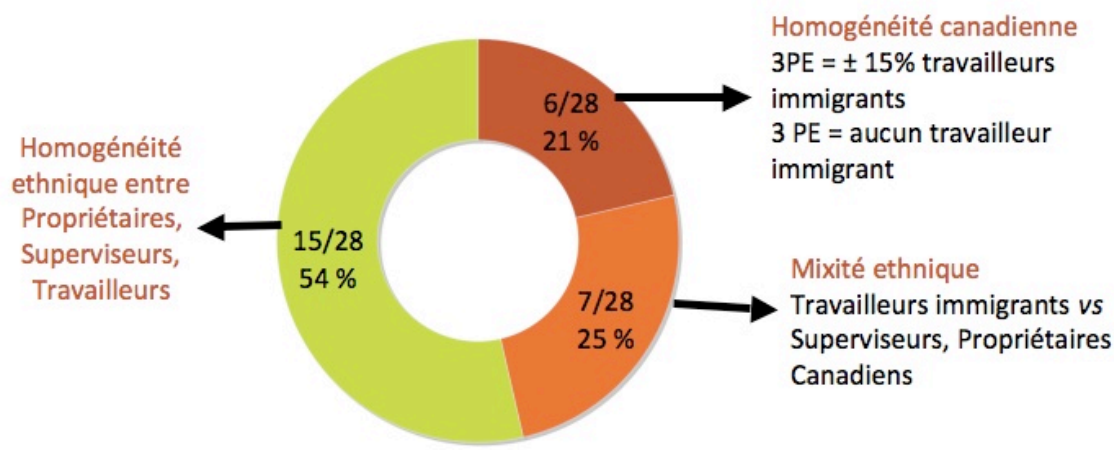


Une tendance s'est dessinée dans les pratiques de SST selon cette composition. D'abord, cette composition semble avoir un impact sur l'implantation d'un comité SST au sein des entreprises. L'absence de comités de SST était notée surtout chez les entreprises mixtes, où les dirigeants et les superviseurs étaient d'origine canadienne alors que les employés étaient issus de l'immigration. Cette tendance était surtout visible dans les entreprises où les employés étaient à 70-80\% issus de l'immigration. En effet, près de la moitié (44\%) des PE n'ayant pas de comité de SST (4/9) se trouvaient dans le groupe des entreprises composées d'une main-d'œuvre mixte. Cela laisse penser que ces PE seraient moins portées à mettre en place un comité de SST.

Les comités de SST des entreprises homogènes sur le plan des origines étaient rarement paritaires: ils étaient composés essentiellement des dirigeants et des superviseurs. Ces comités avaient été créés récemment, depuis moins de trois ans, et fonctionnaient sans objectifs précis : ils répondaient aux normes et aux plaintes, mais ne s'inscrivaient pas dans l'esprit d'une culture préventive et paritaire de SST.

L'homogénéité des origines au sein des PE, bien qu'elle ait été importante, n'est pas surprenante. Elle est la manifestation des réseaux d'insertion sur le marché du travail des immigrants. La communauté ethnique est un réseau privilégié pour trouver un premier emploi. Toutefois, dans notre étude, ce réseau d'insertion sur le marché du travail semblait prolonger la durée d'emploi au-delà des aspirations initiales des travailleurs. Contrairement à ce que nous présumions au départ, les travailleurs immigrants des PE de l'échantillon n'étaient pas immigrants récents. La moitié d'entre eux étaient au Canada depuis plus de 10 ans (figure 3) et $59 \%$ avaient plus de 5 ans d'ancienneté dans l'entreprise.

Figure 2 Pays de naissance et durée de séjour au Canada de la main-d'œuvre des PE de l'échantillon selon les paliers hiérarchiques

Travailleurs

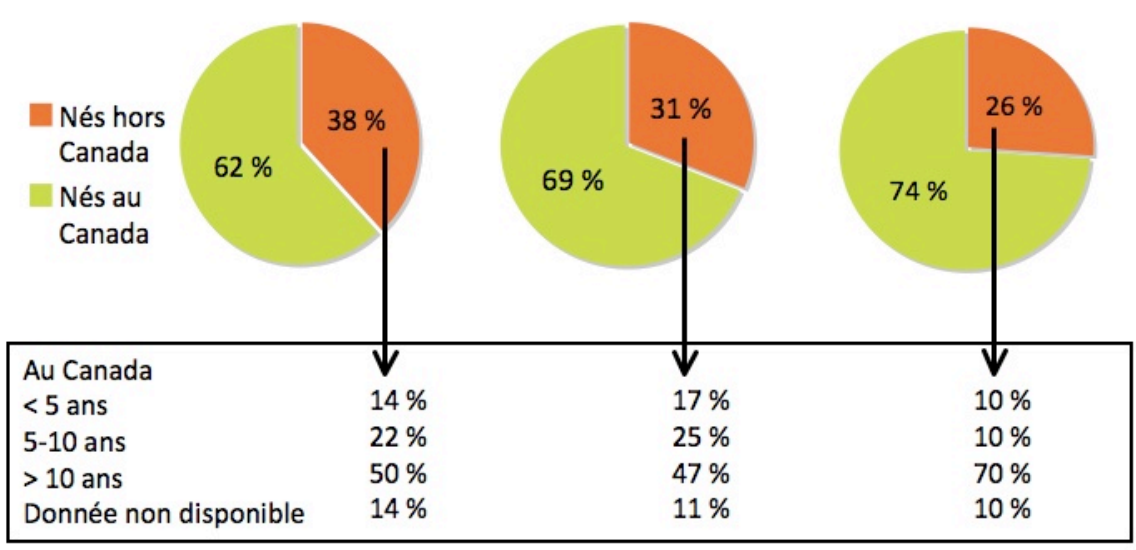

Il est donc faux de penser que les travailleurs immigrants ne sont que de passage dans les PE qui les embauchent. Le collectif de travail composé de collègues issus de la même communauté et parlant la même langue est probablement un élément de stabilisation au sein de l'entreprise qui retient les travailleurs immigrants dans un emploi pour lequel ils sont souvent surqualifiés.

\section{Participation des travailleurs immigrants selon les responsables de SST des petites entreprises}

La majorité $(21 / 28)$ des représentants de SST de notre échantillon assumaient une double et même une triple fonction, par exemple : directeur général et responsable de SST, ou responsable des ressources humaines (RH) et de SST. Une seule entreprise, une fonderie, filiale d'une entreprise internationale, avait embauché un répondant dont l'unique fonction était la coordination de la SST. Lorsque le représentant de la SST est un immigrant, il est dans tous les cas le dirigeant de l'entreprise. Environ le tiers (36 \%) des répondants de SST des entreprises avaient 
suivi une formation en SST au Canada. Cette formation avait été acquise dans le cadre d'un certificat de $1^{\mathrm{er}}$ ou de $2^{\mathrm{e}}$ cycle en SST ou dans le cadre d'études de $2^{\mathrm{e}}$ cycle en gestion.

\section{Participation des travailleurs immigrants au sein du comité de SST}

La majorité (19/28) des entreprises de notre échantillon avaient un comité de SST. Dans les entreprises embauchant une forte proportion d'immigrants, le comité était rarement paritaire. La seule entreprise ( $\mathrm{n}^{\circ} 20$ ) dotée d'un comité paritaire composé essentiellement de travailleurs immigrants (argentins, italiens, haïtiens) a vécu une expérience unique: le comité s'est fait accompagner pendant plus de deux ans par une association sectorielle paritaire (ASP), les aidant à se constituer, à assigner des rôles à chacun des membres du comité, à organiser des formations afin de développer leurs compétences et à procéder à l'analyse des risques au sein de l'entreprise. Au terme de ces deux années d'efforts, le fonctionnement du comité a été perturbé par la crise financière du secteur de production et les revendications syndicales des employés menacées par les licenciements.

De façon générale, les répondants de SST, qui représentaient généralement l'employeur, déploraient la nonparticipation des travailleurs au comité et aux activités de prévention. De plus, on note qu'un grand nombre d'employeurs et de travailleurs ne comprennent pas le principe du paritarisme, les comités de SST formés uniquement de représentants de l'employeur étant un reflet de cette incompréhension.

\section{Formation et initiation à la tâche des travailleurs immigrants}

Des 19 entreprises embauchant des travailleurs immigrants, la moitié n'offrait pas d'orientation structurée à l'embauche. Les pratiques de compagnonnage ou de jumelage constituent souvent un moyen pour superviser les nouveaux employés. Le compagnon originaire du même pays ou maîtrisant les mêmes langues supervise pendant quelques heures ou quelques jours le travail du nouvel employé.

Dans une confiserie $\left(n^{\circ} 36\right)$, la directrice de la production, qui cumulait la direction des ressources humaines et la coordination de la SST, avait constaté que tous ses nouveaux employés hispanophones (60 \%) n'avaient rien saisi des consignes de SST lors de leur initiation. Devant l'insatisfaction de cette initiation, prodiguée par une firme externe, son entreprise décide alors de mandater l'un de ses employés hispanophones parmi les plus anciens pour initier les nouveaux employés et assurer les mises à jour des formations en SST, et ce, pour tout le personnel, composé à $80 \%$ d'immigrants.

Ainsi, certaines entreprises qui embauchent majoritairement des immigrants développent leur propre compétence pour former leurs travailleurs. Cette initiative est surtout vraie dans les entreprises mixtes, où les dirigeants et les superviseurs sont d'origine canadienne et les travailleurs immigrants.

\section{Arguments défavorables à la SST}

Les responsables de la SST ont été interrogés sur les arguments favorables et défavorables à la SST entendus dans leur entreprise, arguments tenus par les dirigeants, les superviseurs ou les travailleurs. Les répondants ont identifié sensiblement les mêmes arguments dans les deux groupes de PE à l'étude. Néanmoins, soulignons que les deux entreprises soutenant le plus grand nombre d'arguments défavorables comptaient plus de $75 \%$ de travailleurs immigrants. Les arguments soulevés étaient: "les mesures de SST demandent trop de temps, ralentissent la production, sont des contraintes et nuisent à l'exécution des tâches ". Les répondants de SST ne semblaient pas porter une attention particulière à ces propos, sauf dans les quelques entreprises où les gestionnaires ayant une formation en gestion des ressources humaines ou en SST. Ces derniers étaient manifestement conscients des effets négatifs de la méconnaissance des travailleurs immigrants à propos du système d'indemnisation, incompréhensions à partir desquelles avaient émergé des idées fausses parmi les travailleurs immigrants.

\section{Mauvaises compréhensions du système d'indemnisation des lésions professionnelles}

Deux entreprises ont souligné les incompréhensions des travailleurs immigrants quant au régime de SST, incompréhensions qui avaient mené à des distorsions pouvant être apparentées à des abus. Dans une entreprise ( $\left.n^{\circ} 106\right)$, les travailleurs immigrants étaient venus s'enquérir auprès de la direction des ressources humaines (DRH), 
lors d'une période de licenciement, du « chômage médical ». Ayant entendu dire que le " chômage médical » était plus avantageux que le " chômage régulier ", les travailleurs voulaient savoir comment choisir leur chômage. La DRH a rapidement fait une mise au point leur expliquant la différence entre ces deux régimes, soit la Commission de la santé et de la sécurité au travail (CSST) et l'assurance-emploi. Dans une autre entreprise ( $\left.n^{\circ} 46\right)$, l'assignation temporaire était considérée comme une "stratégie de l'employeur pour les priver de leur congé à la maison ». La DRH de cette entreprise a jugé opportun de charger un inspecteur de la CSST d'expliquer aux travailleurs immigrants les fondements du régime.

Dans ces deux entreprises, les incompréhensions des travailleurs démontrent que la culture de SST est loin d'être acquise par tous. L'environnement professionnel dans lequel évoluent les employés et les employeurs ainsi que la capacité des entreprises à renouveler la main-d'œuvre contribue à cette dynamique de prise en charge de la SST au-delà des éléments structurels et organisationnels de l'entreprise. Malheureusement, ces éléments, qui font obstruction à la dynamique de la prise en charge autonome de la SST au sein des PE où il y a homogénéité de référents culturels distincts ou mixité, ne font jamais l'objet d'une attention particulière de la part des professionnels de SST.

\section{Participation des travailleurs immigrants selon les professionnels de santé au travail des CLSS}

Les équipes de santé au travail des deux territoires participant à l'étude comptaient respectivement une trentaine de professionnels: médecins, infirmières et techniciens en hygiène du travail. Au total, 26 professionnels de SST ont participé aux entretiens. Pour eux, il a été difficile de se prononcer sur la participation des travailleurs immigrants aux mesures de SST. Néanmoins, deux observations importantes peuvent être faites: les travailleurs immigrants font preuve d'un grand silence et l'attitude des dirigeants ne favorise pas toujours la participation des travailleurs.

Plusieurs professionnels de SAT ont constaté que les travailleurs immigrants exécutent leurs tâches sans exprimer la moindre récrimination. L'idée d'être loyal envers leur employeur, les barrières de la langue et surtout la peur de perdre leur lien d'emploi freinent toute forme de revendication. Ces commentaires ont été relevés, par exemple, dans une entreprise $\left(n^{\circ} 02\right)$ où $90 \%$ des travailleurs sont immigrants et où les trois quarts sont originaires d'Inde, tout comme le sont les dirigeants et les superviseurs.

D'autres observations remettent en question l'attitude des dirigeants dans certaines entreprises (exemples : $n^{\circ} 05$ et $\mathrm{n}^{\circ}$ 56). Eux-mêmes immigrants, ils avaient écarté les travailleurs immigrants des projets de SST. Les rapports d'autorité hiérarchique faisaient en sorte que les travailleurs ne pouvaient pas se prononcer sur les risques et sur les mesures correctrices. En fonction de leur conception de l'autorité et de la responsabilité, les dirigeants avaient endossé toute la responsabilité et assumaient tous les rôles au niveau de la gestion de la SST. Or il s'est avéré qu'ils n'avaient ni les compétences ni le temps pour le faire correctement.

\section{Participation des travailleurs immigrants selon les travailleurs}

Des 28 entreprises de l'échantillon, 19 ont participé à ce volet. Bien que le taux de participation des entreprises ait été de $68 \%$, le taux de participation des travailleurs a été de $30 \%$, soit 181 questionnaires complétés sur 610 (nombre total des employés des 19 entreprises). De nombreux efforts ont été déployés afin d'obtenir la plus large participation des travailleurs à cette cueillette de données. D’abord, chaque PE ayant décliné notre invitation a été relancée, des appels personnalisés ont été faits afin d'expliquer l'importance d'avoir le maximum de travailleurs participants. De plus, à la suggestion des employeurs, le questionnaire a été traduit en anglais, en arabe et en espagnol. Malgré ces efforts, peu de questionnaires en version arabe et espagnole nous ont été retournés. Les travailleurs ayant répondu à ce questionnaire sont majoritairement des hommes (69\%) âgés de 45 ans et plus (61\%). On note que les travailleurs immigrants sont plus scolarisés: $55 \%$ des travailleurs qui ont fait plus de 11 années d'études sont des immigrants $(n=28)$ (figure 3 ). 
Figure 3 Scolarité des travailleurs (en années d'études complétées)

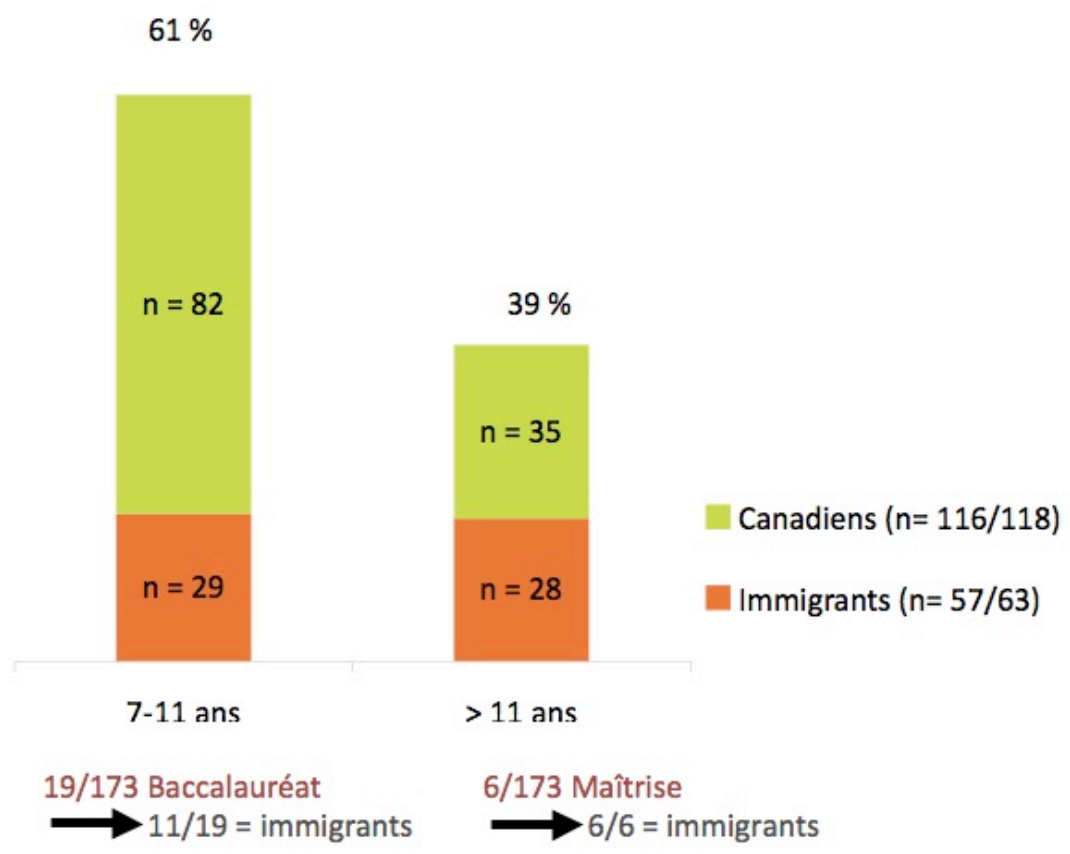

Un test du $\chi^{2}$ a été établi sur les réponses négatives obtenues aux 23 éléments de pratiques minimales de SST dans les entreprises selon la variable de l'origine immigrante ou non immigrante des travailleurs. Le seuil de test statistique a été établi $(p<0,05)$, indiquant que les travailleurs immigrants ont des réponses statistiquement différentes de ceux des Canadiens ou des résultats méritant une attention (tableau 2). Seuls deux résultats (éléments 2 et 10 ) ont atteint ce seuil de signification $(p<0,05)$. Toutefois, nous avons retenu les résultats de 11 éléments dont le seuil de signification se situait entre 0,1 et 0,05 , ce qui indique une tendance non négligeable (éléments $1,3,6,8,9,12,15,17,18,19$ et 22), et pour lesquels des ratios se situant entre 1,5 et 5,5, ce qui indique que les travailleurs immigrants bénéficient moins des mesures de SST prescrites dans leur entreprise. D’autres résultats (éléments 4, 5, 7 et 20) indiquent que certaines pratiques sont déficientes pour tous les travailleurs.

Les résultats significatifs au test du $\chi^{2}(p<0,05)$ démontrent que les travailleurs immigrants ne savaient pas $s^{\prime}$ il $y$ avait un comité de SST dans leur entreprise (élément 2) et ne connaissaient pas les personnes à qui rapporter leur lésion en cas d'accident ou de maladie (élément 10), et ce, malgré le fait qu'ils occupaient généralement ces emplois depuis plusieurs années. D'autres résultats, non statistiquement significatifs $(p>0,05$ et $<0,1)$, indiquent que certaines pratiques auraient tendance à être de 1,5 à 5,5 fois plus défavorables aux travailleurs immigrants qu'aux travailleurs d'origine canadienne. Parmi elles, il y a le manque d'information sur le régime de protection des travailleurs victimes de lésions professionnelles (élément $1: 21 \%$ contre $9 \%$ ), l'absence d'initiation à l'embauche (item 3 : $53 \%$ contre $33 \%$ ), la méconnaissance des règles et procédures de sécurité (élément 6 : $30 \%$ contre $18 \%$ ); l'incapacité à identifier les risques (élément 8: $30 \%$ contre 12\%), l'absence de procédures à suivre en cas d'urgence (élément $9: 12 \%$ contre $5 \%$ ) ou en cas d'incendie (élément 15: $13 \%$ contre $5 \%$ ), ne pas saisir l'intérêt d'informer son employeur même si la blessure est mineure (élément 12 : $19 \%$ contre 8). Les équipements de protection individuelle ont tendance à être moins accessibles aux travailleurs immigrants (élément $17: 25 \%$ contre $12 \%$ ) et lorsqu'ils peuvent en disposer ils semblent moins enclins à les utiliser (élément $18: 15 \%$ contre $4 \%$ ). Les mesures de retour progressif ou d'allègement de la tâche à la suite d'une lésion ont tendance à être moins accessibles aux travailleurs immigrants (élément 19: $18 \%$ contre 9\%). Enfin, les travailleurs immigrants ne semblent pas savoir si leur employeur dispose d'un plan d'entretien des machines et des outils (élément 22 : $15 \%$ contre $6 \%$ ). Le programme pour les travailleuses enceintes semble méconnu des travailleuses immigrantes (élément 23), mais ce résultat indique peut-être une confusion chez les répondants majoritairement masculins, parce qu'il y a peu ou pas de femmes dans leur milieu de travail. 
Tableau 2 Résultats négatifs aux pratiques minimales de santé et de sécurité au travail dans les entreprises

\begin{tabular}{|c|c|c|c|c|}
\hline $\begin{array}{l}\text { NE CONNAIT PAS ces pratiques dans l'entreprise qui } \\
\text { l'embauche.... }\end{array}$ & $\begin{array}{c}\text { Travailleurs } \\
\text { nés au } \\
\text { Canada } \\
(\mathrm{n}=118)\end{array}$ & $\begin{array}{l}\text { Travailleurs } \\
\text { nés hors } \\
\text { Canada } \\
(n=63)\end{array}$ & Ratio & Seuil \\
\hline $\begin{array}{l}\text { 1. Information sur le régime de protection des travailleurs } \\
\text { victimes d'accident ou de maladie professionnelle }\end{array}$ & $9 \%(10)$ & $21 \%(13)$ & 2,34 & 0,07 \\
\hline 2. Présence d'un comité ou d'un représentant de SST & $9 \%(11)$ & $24 \%(16)$ & 2,67 & 0,05 \\
\hline 3. Initiation au poste de travail lors de votre embauche & $32 \%(38)$ & $52 \%(33)$ & 1,63 & 0,09 \\
\hline 4. Information sur les pratiques SST lors de l'initiation & $32 \%(37)$ & $34 \%(21)$ & 1,10 & 0,34 \\
\hline 5. Formation reçue en SST depuis l'embauche & $33 \%(38)$ & $53 \%(33)$ & 1,61 & 0,11 \\
\hline 6. Règles ou procédures de sécurité à suivre & $18 \%(22)$ & $30 \%(19)$ & 1,67 & 0,08 \\
\hline 7. Information sur les produits chimiques utilisés (SIMDUT) & $36 \%(42)$ & $37 \%(23)$ & 1,03 & 0,80 \\
\hline $\begin{array}{l}\text { 8. Capacité de nommer un risque d'accident ou de maladie } \\
\text { dans l'entreprise }\end{array}$ & $12 \%(15)$ & $30 \%(19)$ & 2,50 & 0,06 \\
\hline 9. Information sur les procédures à suivre en cas d'urgence & $5 \%(6)$ & $12 \%(8)$ & 2,43 & 0,09 \\
\hline 10. Personnes à informer en cas d'accident (ne sais pas) & $7 \%(9)$ & $23 \%(15)$ & 3,29 & 0,05 \\
\hline 11. Personnes à informer en cas d'incident (ne sais pas) & $6 \%(7)$ & $7 \%(4)$ & 1,00 & 1,10 \\
\hline $\begin{array}{l}\text { 12. Prévenir l'employeur lorsqu'il y a blessure mineure sans } \\
\text { absence }\end{array}$ & $8 \%(9)$ & $19 \%(12)$ & 2,00 & 0,07 \\
\hline 13. Exercer un droit de refus lorsque la tâche est dangereuse & $5 \%(5)$ & $7 \%(4)$ & 1,40 & 1,01 \\
\hline 14. Participation à une enquête à la suite d'un accident & $2 \%(3)$ & $11 \%(7)$ & 5,50 & 1,06 \\
\hline 15. Connaissance des mesures d'urgence en cas d'incendie & $5 \%(6)$ & $13 \%(8)$ & 2,60 & 0,07 \\
\hline $\begin{array}{l}\text { 16. Aide apportée aux travailleurs blessés pour remplir leur } \\
\text { déclaration d'accident }\end{array}$ & $17 \%(21)$ & $31 \%(20)$ & 1,83 & 0,11 \\
\hline 17. Disposer d'un équipement de protection individuelle & $12 \%(15)$ & $25 \%(16)$ & 2,09 & 0,09 \\
\hline 18. Porter l'équipement de protection individuelle & $4 \%(5)$ & $15 \%(10)$ & 3,75 & 0,06 \\
\hline $\begin{array}{l}\text { 19. Bénéficier d'un retour progressif ou d'un allègement de } \\
\text { tâche après une lésion }\end{array}$ & $9 \%(11)$ & $18 \%(18)$ & 2,00 & 0,08 \\
\hline 20. Programmes d'activités de prévention & $34 \%(40)$ & $38 \%(24)$ & 1,12 & 0,66 \\
\hline $\begin{array}{l}\text { 21. Tableau ou relevé périodique du nombre d'accidents et } \\
\text { de jours d'absence }\end{array}$ & $51 \%(60)$ & $65 \%(41)$ & 1,28 & 0,78 \\
\hline 22. Machinerie et outillage bien entretenus & $6 \%(8)$ & $15 \%(10)$ & 2,50 & 0,06 \\
\hline $\begin{array}{l}\text { 23. Programme d'affectation ou retrait de la travailleuse } \\
\text { enceinte ou qui allaite (ne s'applique pas : } 40 \% \text { et } 2 \% \text { ) }\end{array}$ & $22 \%(26)$ & $42 \%(26)$ & 1,91 & 0,12 \\
\hline
\end{tabular}

\begin{tabular}{|c|c|c|}
\hline $\begin{array}{c}\text { Seuil significatif } \\
p<0,05\end{array}$ & Tendance & Aucune signification \\
& $0,1<p>0,05$ & statistique \\
\hline
\end{tabular}

II est préoccupant que certaines pratiques préventives de SST soient déficientes, que ce soit auprès des travailleurs immigrants ou non immigrants, étant donnée la gravité des situations qui peuvent en découler. Plus du tiers des travailleurs, indépendamment de leur origine, ont dit n'avoir bénéficié d'aucune information que ce soit en SST lors de leur embauche (élément $4: 34 \%$ et $32 \%$ ) ou en cours d'emploi (élément $5: 33 \%$ et $53 \%$ ), ou bien sur les produits chimiques employés (élément $7: 36$ et $37 \%$ ). Il en est de même avec les programmes de prévention, connus ni des travailleurs d'origine canadienne ni de ceux issus de l'immigration (élément 20 : $34 \%$ et 38 \%). Pour finir, retenons que même si les résultats ne sont pas statistiquement significatifs, les travailleurs immigrants participaient moins souvent aux enquêtes à la suite d'un accident (élément 14: $11 \%$ contre $2 \%$ ). 
Ces résultats confirment les observations réalisées tant par les représentants de SST des PE que par les professionnels de la SAT : les travailleurs immigrants sont moins bien formés et informés en matière de SST et ils participent peu ou pas aux mesures préventives et correctrices. En fait, le taux de participation des travailleurs immigrants au questionnaire autoadministré indique qu'ils ne se sentent même pas interpellés par une étude consacrée à leur condition.

\section{Pratiques exemplaires susceptibles d'inciter les travailleurs immigrants à participer}

Au terme de l'étude, sept PE se sont démarquées pour avoir introduit des pratiques incitatives à la participation des travailleurs immigrants. Chacune avait des objectifs distincts et des stratégies différentes :

- Former les nouveaux travailleurs en se basant sur un système de compagnonnage entre travailleurs de même origine ou s'exprimant dans une langue maternelle commune;

- Lors de l'embauche ou lors d'un changement de méthodes de travail, initier les travailleurs à la tâche en présence d'un interprète;

- Offrir des cours de francisation aux travailleurs immigrants pendant une période de ralentissement de la production;

- Offrir des séances d'information aux travailleurs immigrants sur le système d'indemnisation, les coûts de cotisation et leur impact sur la rentabilité de l'entreprise;

- Accompagner les travailleurs immigrants dans la création d'un comité de SST, définir ses tâches, initier les membres à l'analyse des risques et implanter des mesures correctrices.

Ces quelques exemples de stratégies adaptées aux travailleurs immigrants ont permis, dans la plupart des cas, une stabilisation de la main-d'œuvre au sein de l'entreprise, et ce, malgré des périodes de ralentissement de production et de crise économique. Ces stratégies ont été mises en œuvre par les dirigeants - bien souvent par le directeur des $\mathrm{RH}$ ou le gestionnaire comptable - et quelques-unes ont été soutenues financièrement par des programmes gouvernementaux d'aide aux entreprises en période de ralentissement économique. Ces sept entreprises, ainsi que les autres, déplorent la non-contribution des instances de SST à l'adaptation de leurs stratégies auprès des travailleurs immigrants et allophones qui composent parfois la majorité de leur maind'œuvre.

\section{Discussion}

À la lumière de la recension des écrits et celle des échanges avec le comité de suivi (comité composé de relayeurs pour le transfert des connaissances vers les communautés de pratique), trois éléments expliqueraient la faible participation des travailleurs immigrants à des mesures de prévention en SST :

- Les travailleurs immigrants s'investissent peu ou pas dans ces emplois d'insertion sur le marché du travail;

- Les employeurs et les superviseurs, eux-mêmes issus de l'immigration, saisissent mal la culture de la SST;

- Les entreprises qui réussissent à impliquer les travailleurs immigrants ont des gestionnaires détenant une formation en SST acquise au Canada.

Selon la première explication, les travailleurs immigrants s'investissent peu ou pas dans ces emplois de PE qui sont au départ des emplois d'insertion sur le marché du travail, des emplois pour lesquels ils sont surqualifiés (Chen, Smith et Musitard, 2010) et qui seraient donc transitoires. Nos résultats ne confirment pas cette hypothèse. La majorité (59\%) des travailleurs immigrants et des travailleurs d'origine canadienne occupent leur emploi depuis plusieurs années, soit depuis plus de cinq ans. Comme le soulignait un répondant de SST d'une PE employant une majorité de femmes originaires des Philippines qui possède des compétences supérieures à celles qui sont exigées par le poste qu'elles occupent, « l'attraction du collectif de travail serait un incitatif à demeurer loyal à ces premiers emplois d'insertion économique ». 
La deuxième explication repose sur la conviction que les employeurs et les superviseurs, en particulier ceux qui sont eux-mêmes issus de l'immigration, saisiraient mal la culture de la SST, notamment à propos de la participation paritaire. Cette hypothèse est confirmée. Ne maîtrisant peu ou pas les lois et les règlements de SST, ils ne créent pas les conditions favorables à la participation des travailleurs immigrants. L'existence de comités de SST non paritaires, coordonnés essentiellement par les gestionnaires (qui s'opposent parfois à toute représentation syndicale) va dans ce sens.

La troisième explication est liée à la formation et au rôle spécifique des responsables internes de SST. En effet, les quelques entreprises qui réussissent à impliquer les travailleurs immigrants sont généralement des entreprises dont les gestionnaires ont une formation en SST, un certificat ou des compétences acquises dans le cadre d'un diplôme en gestion au Canada. Cela est également vrai pour les superviseurs qui sont nés à l'étranger. Les principes de parité et de participation volontaire des travailleurs font alors partie intégrante de leur mode de gestion. Néanmoins, même dans ce dernier cas, les dirigeants et les superviseurs doivent inciter les travailleurs immigrants à s'impliquer, puisque cette initiative ne vient jamais des travailleurs eux-mêmes.

Certes, cette étude exploratoire présente des limites méthodologiques. Notre échantillon de 28 PE est petit et il pourrait facilement être augmenté et inclure des entreprises de secteurs de production non prioritaires (services, transformation alimentaire, etc.). Les entreprises pourraient être sollicitées sur une autre base que celle du registre des CSSS, mais nous introduirions un biais de sélection en incluant un plus grand nombre d'entreprises peu touchées par des mesures de surveillance en SST.

Nous pourrions constituer un échantillon de travailleurs par grappe, par entreprise. Pour chaque entreprise, nous pourrions par exemple viser un taux de participation des travailleurs de $40 \%$. Cependant, il faudrait pour cela associer les répondants à leur entreprise, ce qui nuirait au principe d'anonymat auquel les travailleurs immigrants semblent très sensibles. Initialement, nous avions proposé d'offrir aux travailleurs immigrants un service d'interprète pour remplir le questionnaire afin d'assurer une meilleure participation. Les dirigeants ont rejeté cette façon de procéder, trouvant qu'elle mobilisait trop de travailleurs sur le temps de travail. Bref, plusieurs considérations méthodologiques pourraient bonifier une telle étude.

Par ailleurs, il serait pertinent d'approfondir un certain nombre de facteurs contribuant à la dynamique de la culture de SST qui expliquerait la non-participation des travailleurs aux mesures de prévention en SST. Nous pensons plus particulièrement aux barrières linguistiques et aux modalités de communication des protocoles de prévention; à l'expérimentation de modules de formation en prévention SST culturellement adaptés; à une étude longitudinale de trajectoires de carrières des travailleurs immigrants et à une compréhension fine des représentations de la notion de santé, de bien-être et de risque au travail. Finalement, il serait important d'explorer l'hypothèse selon laquelle les travailleurs immigrants s'investissement moins et participent moins aux mesures de SST parce que les emplois qu'ils occupent sont en principe temporaires, mais finissent par être permanents.

\section{Conclusion}

À la lumière des résultats de cette étude, nous recommandons aux instances de SST et aux établissements qui ont le mandat d'accompagner les entreprises dans leur prise en charge autonome de la SST de faire en sorte :

- Que tous les travailleurs et travailleuses récemment immigrés reçoivent une formation sur leurs droits, leurs obligations et leurs recours en matière de SST.

- Que les propriétaires des PE (<50 travailleurs) puissent bénéficier d'une formation sur les lois, les règlements et les obligations des employeurs en matière de SST ainsi que d'une mise à jour lors des changements.

- Qu'il y ait une offre de service qui soit faite aux PE pour soutenir et accompagner le développement des comités de SST afin de définir le mandat de leur comité, de partager les responsabilités entre les membres du comité, de développer les compétences reliées à chacune de ces responsabilités et d'implanter des stratégies pour assurer la participation des travailleurs, et particulièrement celle des travailleurs immigrants.

Alterstice - Revue Internationale de la Recherche Interculturelle, vol. 2, $n^{\circ} 2$ 
- Que les travailleurs immigrants et allophones bénéficient de formations en SST et d'une initiation à la tâche lors de leur embauche ainsi que de formations adaptées à leur capacité de saisir correctement et sans ambiguïtés les consignes de sécurité. Cette formation devrait être traduite et adaptée si, culturellement, les concepts, les termes ou les équipements sont différents.

- De créer une banque multilingue de formateurs qui pourraient offrir ces services aux entreprises ayant des besoins spécifiques de formation (ex : chariot élévateur, SIMDUT, etc.).

- D'introduire des outils pour inciter les PE à prendre en charge de façon autonome leur programme spécifique de santé pour les PE qui embauchent dans une proportion importante des travailleurs immigrants. II faudrait également faire en sorte que les obstacles culturels et linguistiques et la méconnaissance des lois de SST soient atténués et surmontés par une approche interculturelle de la SST.

- De développer des projets pilotes en lien avec ces recommandations, projets qui feraient l'objet d'une recherche évaluative (implantation, impacts et effets).

L'embauche de travailleurs immigrants n'est pas un phénomène marginal. Elle représente plutôt une solution au problème chronique d'embauche dans les $P E$, dans lesquelles les savoir-faire sont néanmoins mal reconnus et souvent moins bien rémunérés.

\section{Références bibliographiques}

Amodu, T. (2008). The determinants of compliance with laws and regulations with special reference to health and safety. A literature review (Report RR-638). London School of Economics and Political Science for the Health and Safety Executive (HSE).

Bouchard, P. (2008). Les entreprises de 11 à 49 employés : portrait de leur réalité linguistique. Québec : Office québécois de la langue française.

Brown, M., Domenzain A. et Villoria-Siegert, N. (2002). California's immigrant workers speak up about health and safety in the workplace. UCLA-LOSH Health \& safety policy brief, december 2002, 1-4.

Champoux, D. et Brun, J.-P. (1999). Prise en charge de la sécurité dans les petites entreprises des secteurs de I'habillement et de la fabrication de produits en métal (Rapport R-226). Montréal : Études et recherches IRSST.

Chicha, M.-T. et Charest, E. (2008). L'intégration économique des immigrés sur le marché du travail à Montréal : politiques et enjeux. Choix IRPP, 14(2), 1-62. www.irpp.org/fr/choices/archive/vol14no2.pdf

Citoyenneté et immigration Canada (2011). Canada faits et chiffres 2011. Aperçu de l'immigration, résidents permanents et temporaires. http://www.cic.gc.ca/francais/pdf/recherche-stats/faits2011.pdf

Citoyenneté et immigration Canada (2012). Rapport annuel au Parlement sur l'immigration. http://www.cic.gc.ca/francais/pdf/pub/rapport-annuel-2012.pdf

Chen, C., Smith, P. et Mustard, C. (2010). The prevalence of over-qualification and its association with health status among occupationally active new immigrants to Canada. Ethnicity and Health, 15(6), 601-619.

Demarco, A. et Georgy, B. (2009). L'avenir du Canada atlantique : Le défi du déclin démographique. Toronto : Fédération canadienne des entreprises indépendantes. http://www.cfib-fcei.ca/cfib-documents/rr3092f.pdf

Demarco, A., McGrath-Gaudet, E. et Hindy, J. (2007). Bâtir un avenir prospère : point de vue des PME sur la productivité au Canada atlantique. Toronto : Fédération canadienne de l'entreprise indépendante. http://www.cfib-fcei.ca/cfib-documents/rr3207f.pdf

Desmarais, L. (2004). Évaluation de l'implantation des comités de santé et de sécurité du travail : une étude de cas multiples réalisée dans les petites et moyennes entreprises au Québec (thèse de doctorat). Université du Québec à Montréal. 
Eakin, J., Lamm, F. et Limborg, H. (2000). International perspective on the promotion of health and safety in small workplace. Dans K. Frick, P. L. Jensen, M. Quinlan et T. Wilthagen (dir.), Systematic occupational health and safety management : perspectives on an international development (p. 227-247). Oxford : Pergamon Press.

Fédération canadienne des entreprises indépendantes (FCEI). (2011). L'immigration et la petite entreprise : une solution aux pénuries de main-d'œuvre et de compétences. www.cfib-fcei.ca/cfib-documents/DIN0529F.pdf

F.E.R.M.E. (2000). Répartition des travailleurs - Secteurs non-agricoles [graphique en ligne]. www.fermequebec.com/DATA/DOCUMENT/430_fr v $\sim$ Repartition_des_travailleurs_-_Secteurs_nonagricoles.pdf

Forcier, M. et Handal, L. (2012). L'intégration des immigrants et immigrantes au Québec (note socio-économique). Montréal : Institut de recherche et d'informations socio-économiques (IRIS). http://www.irisrecherche.qc.ca/publications//\%E2\%80\%99integration-des-immigrants-et-immigrantes-au-quebec

Gravel, S., Brodeur, J.-M., Champagne, F., Lippel, K., Fournier, M., Boucheron, L. et Patry, L. (2006). Critères pour apprécier les difficultés d'accès à l'indemnisation des travailleurs victimes de lésions professionnelles [en ligne]. Perspectives interdisciplinaires sur le travail et la santé (PISTES), 8(2), 15 p. http://www.pistes.uqam.ca/v8n2/articles/v8n2a6.htm

Gravel, S., Brodeur, J.-M., Champagne, F., Lippel, K. et Vissandjée, B. (2007). Incompréhension des travailleurs immigrants victimes de lésions professionnelles de leurs difficultés d'accéder à l'indemnisation. Migration et santé, 131(2), 11-42.

Gravel, S., Rhéaume, J. et Legendre, G. (2011). Strategies to develop and maintain occupational health and safety measures in small businesses employing immigrant workers in metropolitan Montreal. International Journal of Workplace Health and Management (IJWHM), 4(2), 164-178.

Handal, L., Viens, P., Hurteau, P. et Hébert, G. (2012). La migration de main-d'œuvre temporaire : ses causes et répercussions. Montréal : Institut de recherche et d'informations socio-économiques (IRIS).

Health and Safety Executive (HSE) (2005). Occupational health and safety support systems for small and medium sized enterprises. A Literature Review (Report RR-410). Berks (RU) : Bomel Limited for the Health and Safety Executive (HSE). www.hse.gov.uk/research/rrpdf/rr410.pdf

Hennebry, J. Preibisch, K. (2010). A model for managed migration? Re-examining best practices in Canada's seasonal agricultural worker program. International Migration, 50(S1), e19-e40.

Henshaw, J. (2002). Testimony : occupational safety and health administration's efforts to protect immigrant workers. Washington DC : Occupational Health and Safety Administration (OSHA), United States Seatle Subcommittee on Employment Safety and Training.

Lachance, A. (2008).Travailleurs immigrants et SST. Un nouveau continent à découvrir. Prévention au travail, 21(3), 7-14.

Lacroix, S. et Pipon, N. (2007). Portrait de la situation des personnes immigrantes actives salariées dans l'ensemble des secteurs d'activité économique pour la région métropolitaine de Montréal et l'île de Montréal : résultats statistiques. Emploi Québec, CAMO immigration et ÉduConseil.

Lansdown, T., Deighan, C. et Brotherton, C. (2007). Health and safety in the small to medium-sized enterprise. Psychosocial opportunities for intervention (Report RR-578). Edinburgh : Heriot Watt University for the Health and Safety Executive (HSE). www.hse.gov.uk/research/rrpdf/rr578.pdf

Levée, V. (2011). Faire connaître la prevention aux travailleurs agricoles étrangers. Prévention au travail, 24(3), 4041.

Kosny, A., Lifshen, M., Maceachen, E., Smith, P., Jafri, G., Neilson, C., Pugliese, D. et Shield, J. (2012). Delicate dances : immigrant workers' experiences of injury reporting and claim filing. Ethnicity and Health, 17(3):267290. 
Mendeloff, J., Nelson, C., Ko, K. et Haviland, A. (2006). Small Businesses and Workplace Fatality Risk - An Exploratory Analysis (TR-371). Kauffman-RAND Center for the Study of Small Business and Regulation.

Premji, S., Duguay, P., Messing, K. et Lippel, K. (2010). Are immigrants, ethnic and linguistic minorities overrepresented in jobs with a high level of compensated risk? Results from a Montréal, Canada study using census and workers' compensation data. American Journal of Industrial Medicine, 53, 875-885.

Quinlan, M. et Mayhew, C. (2000). Precarious employment, work re-organisation and the fracturing of OHS management. Dans K. Frick, P. L. Jensen, M. Quinlan et T. Wilthagen (dir.), Systematic occupational health and safety management : perspectives on an international development (p. 175-198). Oxford :Pergamon Press.

Simard, M., Carpentier-Roy, M. C., Marchand, A.S. et Ouellet, F. (2002). Étude exploratoire des dynamiques préventives en santé au travail dans les petits établissements (Rapport R-320). Montréal : Études et recherches IRSST.

Smith, P. et Mustard, C. (2009). Comparing the risk of work-related injuries between immigrants to Canada, and Canadian-born labour market participants. Occupational and Environmental Medicine, 66(6), 361-367.

Smith, P. et Mustard, C. (2010). The unequal distribution of occupational health and safety risks among immigrants to Canada compared to Canadian-born labour market participants: 1993-2005. Safety Science, 48(10), 12961303.

Socialstyrelsen. (1995). Invandrares hälsa och sovciala förhållanden (SoS-rapport 1995:5). Stockholm. [cité par Wren, K. et Boyle, P. (2001). Migration and work-related health in Europe - A literature review. Stockholm : National Institute for Working Life / SALTSA].

Statistique Canada. (2009). Programme des travailleurs étrangers temporaires. Statistiques sur les avis relatifs au marché du travail. Statistiques annuelles archivées 2005-2008 [en ligne]. http://www.hrsdc.gc.ca/fra/competence/travailleurs_etrangers/stats/archive/annuel20052008/tableau9a.shtml

Underhill, E. et Quinlam, M. (2011) How precarious employment affects health and safety at work: the case of temporary agency workers. Relations industrielles, 66(3), 397-421.

Workers compensation board of Manitoba (2008). Developing safety resources for immigrant workers: the Manitoba immigrants' safety initiative, WCB Update, june 2008, 1.

http://www.wcb.mb.ca/sites/default/files/june-08-update.pdf 